32 Revista Latinoamericana de la Papa 20 (1): 32 - 45

ISSN: $1853-4961$

http://www.papaslatinas.org/ojs/index.php/index/oai

\title{
Efecto de la interacción genotipo por ambiente sobre los contenidos de Hierro, Zinc y Vitamina C en Genotipos de Papa (Solanum sp.)
}

\author{
J. Rivadeneira ${ }^{1 / *}$, D. Ortega ${ }^{2}$, V. Morales ${ }^{3}$, C. Monteros ${ }^{1}$, X. Cuesta ${ }^{1}$
}

Recibido: $22 / 02 / 2016$

Aceptado: 09/03/2016

Accessible en linea: Junio 2016

\section{Resumen}

Con el objetivo de determinar el efecto de la interacción genotipo por ambiente (GEI) sobre el contenido de hierro (Fe), zinc ( $\mathrm{Zn})$ y vitamina $\mathrm{C}(\mathrm{VC})$ en papa, se establecieron ensayos en cuatro ambientes de la Sierra Ecuatoriana. Se evaluó el rendimiento, los contenidos de Fe, Zn, VC y se estimó la heredabilidad en el sentido amplio $\left(\mathrm{H}^{2}\right)$. Como resultado se obtuvo un efecto significativo de los factores en estudio para los diferentes contenidos. $\mathrm{La} \mathrm{H}^{2}$ fue de $0.32,0.20,0.35$ y 0.80 para $\mathrm{Fe}, \mathrm{Zn}, \mathrm{VC}$ y rendimiento respectivamente. Esta investigación mostró genotipos con altos contenidos de $\mathrm{Fe}\left(>43 \mu \mathrm{g} \cdot \mathrm{g}^{-1}\right.$ de peso seco promedio) como INIAP-Victoria, Superchola y los clones 98-38-12 y 07-32-1, en cuanto al $\mathrm{Zn}\left(>18 \mu \mathrm{g}\right.$. g ${ }^{-1} \mathrm{de}$ peso seco promedio) los clones 07-40-1, 07-32-1, 07-46-8 y la variedad INIAP-Victoria presentaron valores altos, mientras que para vitamina $\mathrm{C}\left(>98 \mathrm{mg} .100 \mathrm{~g}^{-1}\right.$ de peso fresco promedio) el clon 07-24-18 presentó el mayor contenido y finalmente los genotipos con rendimientos superiores a 40 t. ha $^{-1}$ fueron el clon 98-2-6 y las variedades INIAP-Estela, INIAP-Fripapa e INIAP-Victoria. Las concentraciones de $\mathrm{Fe}, \mathrm{Zn}$ y $\mathrm{VC}$ fueron significativamente afectadas por el genotipo, el ambiente y su interacción. Sin embargo, la mayor variación se debió al efecto del ambiente la cual estuvo principalmente asociada con la fertilización, humedad y $\mathrm{pH}$ del suelo.

Palabras clave adicionales: $\mathrm{Fe}, \mathrm{Zn}$, vitamina $\mathrm{C}$, heredabilidad.

\section{Effect of the interaction genotype by environment in the content of Iron, Zinc and Vitamin $\mathrm{C}$ in potato tuber (Solanum sp.)}

\section{Summary}

In order to determine the effect of the interaction genotype by environment (GEI) in the content of iron $(\mathrm{Fe})$, zinc $(\mathrm{Zn})$ and vitamin $\mathrm{C}(\mathrm{VC})$ in potato tubers, field experiments were established in four environments of the Ecuadorian Highlands. Content of, Fe, Zn, VC, yield and broad-sense heritability $\left(\mathrm{H}^{2}\right)$ were evaluated. As result the $\mathrm{H}^{2}$ for $\mathrm{Fe}, \mathrm{Zn}, \mathrm{VC}$ content and yield was $0.32,0.20,0.35$ and 0.80 , respectively. This research showed genotypes with high content of $\mathrm{Fe}$ (> $43 \mathrm{mg} \mathrm{g}^{-1}$ dry weight) as Superchola, INIAP-Victoria and the clones 98-3812 and 07-32-1, while for $\mathrm{Zn}\left(>18 \mu \mathrm{g} \mathrm{g}^{-1}\right.$ of dry weight) the clones 07-40-1, 07-32-1 and INIAP-Victoria had the highest content, whereas for VC (>98 mg.100 g ${ }^{-1}$ fresh weight) the clon 07-24-18 had the highest value. Finally the genotypes with higher yields $>40 \mathrm{t}^{\text {. ha }} \mathrm{h}^{-1}$ were the clon 98-2-6 and the varieties INIAP - Estela, INIAP - Fripapa and INIAP -Victoria.

Autor para correspondencia. Correo electrónico: jorge.rivadeneira@iniap.gob.ec

Instituto Nacional de Investigaciones Agropecuarias (INIAP), Estación Experimental Santa Catalina, Panamericana Sur km1 Quito, Ecuador.

2 Universidad Central de Ecuador. Quito - Ecuador.

3 Escuela Superior Politécnica de Chimborazo-ESPOCH. Chimborazo-Ecuador. 
The Fe, Zn and VC content were significantly affected by genotype, environment and their interaction. However, the greatest variation was due to the effect of the environment, it was mainly related with the fertilization content, soil moisture and $\mathrm{pH}$.

Additional Keywords: Fe, Zn, vitamin C, heritability.

\section{Introduction}

La papa (Solanum tuberosum L.) el trigo, el arroz y maíz son los principales cultivos consumidos en el mundo (http://faostat.fao.org). La papa proporciona a las personas no sólo hidratos de carbono (almidón y azúcares) sino también otros compuestos esenciales para la salud humana. El tubérculo contiene carbohidratos, tiene un buen balance de aminoácidos, vitamina B6, B1 y folato (Storey, 2007). Un tubérculo de $200 \mathrm{~g}$ de papa fresca proporciona a una persona entre 17 al $18 \%$ del requerimiento diario de hierro y del 5 al 13\% de Zinc (White et al., 2009).

La vitamina $\mathrm{C}$ o ácido ascórbico es la principal vitamina en el tubérculo de papa. Se estima que $200 \mathrm{~g}$ de tubérculos cocidos proporcionan hasta el $47 \%$ de la cantidad diaria recomendada (Haase, 2008). Estudios realizados indican que las concentraciones más altas de vitamina $\mathrm{C}$ en los tubérculos comienzan en el último periodo de desarrollo del cultivo antes del inicio de senescencia y la disminución ocurre en la maduración y almacenamiento (Love y Pavek, 2008).

La composición química de los tubérculos de papa es variable y está controlado principalmente por factores genéticos, factores ambientales como suelo, clima, agua, manejo del cultivo y madurez de los tubérculos, así como los procesos de cocción y el almacenamiento que afectan la composición química de la papa y como consecuencia su valor nutricional (Bonierbale et al., 2008). La presente investigación tuvo como objetivo determinar el efecto de la interacción genotipo por ambiente (GEI) sobre los contenidos de $\mathrm{Fe}, \mathrm{Zn}$ y vitamina $\mathrm{C}$ en clones y variedades mejoradas de papa.

\section{Materiales y métodos}

\section{Ubicación}

La investigación se llevó a cabo en cuatro ambientes de la Sierra del Ecuador: en Aláquez provincia de Cotopaxi a 2959 msnm con una temperatura y precipitación promedio anual de $12.6{ }^{\circ} \mathrm{C}$ y $450 \mathrm{~mm}$ respectivamente; en la Estación Experimental Santa Catalina (EESC) del INIAP ubicada en Cutuglahua provincia de Pichincha a $3058 \mathrm{msnm}$ con una temperatura y precipitación promedio anual de $12.0 \quad{ }^{\circ} \mathrm{C} \quad \mathrm{y} \quad 1432.0 \mathrm{~mm}$ respectivamente; en Tunshi provincia de Chimborazo ubicada a $2738 \mathrm{msnm}$, con una temperatura y precipitación promedio anual de $13.2{ }^{\circ} \mathrm{C}$ y $421.2 \mathrm{~mm}$ y en Chiquicha provincia de Tungurahua a 2519 msnm con una temperatura y precipitación promedio anual de $17.0{ }^{\circ} \mathrm{C}$ y $750.0 \mathrm{~mm}$ respectivamente.

\section{Material Vegetativo}

Catorce genotipos de papa se evaluaron, de los cuales nueve fueron clones del programa de mejoramiento y cinco variedades mejoradas (Tabla 1). 
Tabla 1. Origen genético de 14 genotipos de papa (Solanum sp.) para evaluación de la GEI sobre los contenidos de Fe y $\mathrm{Zn}$ en cuatro ambientes.

\begin{tabular}{ll}
\hline \multicolumn{1}{c}{ Genotipos } & \multicolumn{1}{c}{ Pedigrí } \\
\hline $97-25-3$ & B x INIAP-Fripapa \\
$98-2-6$ & INIAP-Gabriela x INIAP-Margarita \\
$98-38-12$ & $95-26-4$ x 96-1-5 \\
$07-46-8$ & $05-18-5 \times$ INIAP-Estela \\
$07-40-1$ & $05-10-4 \times$ INIAP-Fripapa \\
$07-32-1$ & BOM532 x HSO213 \\
$07-32-15$ & BOM532 x HSO213 \\
$07-28-2$ & ASO861 x BOM532 \\
$07-24-18$ & HSO198 x ASO861 \\
INIAP-Natividad & INIAP-Gabriela x S. phureja x S. pausissectum \\
INIAP-Estela & Superchola x S. phureja x S. pausissectum \\
INIAP-Victoria & INIAP-Gabriela x INIAP-Fripapa \\
INIAP-Fripapa & Bulk México x 378I58,721 x I-I039 \\
Superchola & Curipamba negra x Solanum demissum x clon \\
& resistente con comida amarilla x chola seleccionada \\
\hline
\end{tabular}

\section{Variables de Estudio}

Las variables registradas fueron rendimiento de tubérculo (RT) en toneladas por hectárea $\left(\mathrm{t} \cdot \mathrm{ha}^{-1}\right)$, contenido de vitamina $\mathrm{C}(\mathrm{VC})$ en $\mathrm{mg} .100 \mathrm{~g}^{-1}$ de peso fresco $(\mathrm{PF})$, hierro $(\mathrm{Fe})$ y zinc $(\mathrm{Zn})$ en $\mu \mathrm{g} \cdot \mathrm{g}^{-1}$ de peso seco (PS).

\section{Preparación de las muestras y determinación de $\mathrm{Fe}$, Zn y Vitamina $\mathrm{C}$}

Para estimar el contenido de Fe y $\mathrm{Zn}$, a la cosecha se tomó una muestra de $0.5 \mathrm{~kg}$ de tubérculos de cada genotipo por repetición y por ambiente, la cual fue lavada, secada y colocada en una funda de papel y enviada al laboratorio del Departamento de Manejo de Suelos y Aguas (DMSA) de la Estación Experimental Santa Catalina (EESC) del INIAP para su análisis. Cada muestra fue lavada con agua destilada, después las muestras fueron secadas en una estufa a $60{ }^{\circ} \mathrm{C}$ por 48 horas. Una vez seca la muestra fue molida utilizando un molino triturador y su posterior tamizado a través de una malla número 40. Para la extracción de Fe y $\mathrm{Zn}$ se pesó $0.25 \pm 0.0002 \mathrm{~g}$ de material vegetal seco colocado en un matraz erlenmeyer de $50 \mathrm{~mL}$. Luego se agregaron $5 \mathrm{~mL}$ de la mezcla de ácido nítrico-perclórico relación 5:1. Posteriormente se colocaron los matraces
Erlenmeyer en una plancha de digestión, precalentada a una temperatura aproximada a $100{ }^{\circ} \mathrm{C}$, se esperaron 15 minutos y se incrementó la temperatura a $150{ }^{\circ} \mathrm{C}$, luego se esperó que los humos pardos del ácido nítrico desaparezcan. $\mathrm{La}$ digestión se consideró completa cuando el digestado fue transparente. Una vez que se enfrió, se añadieron $25 \mathrm{~mL}$ de agua desmineralizada, agitando la solución para lavar los lados del frasco y para filtrar se utilizó papel filtro. Finalmente se procedió a realizar las lecturas correspondientes de cada muestra usando el equipo ICP (Espectroscopia de emisión por acoplamiento de plasma inductivo) de marca Perkin Elmer modelo Óptima 5300.

Para VC se tomó una muestra de $1.0 \mathrm{~kg}$ de tubérculos de cada genotipo por repetición, las muestras fueron lavadas, secadas y colocadas en fundas de papel y enviadas al laboratorio del Departamento de Nutrición y Calidad (DNC) de la EESC del INIAP para su análisis. Se utilizó el método reflectométrico de la MERCK, equipo Reflectómetro RQ flex 16970, MERCK.

\section{Análisis Estadístico}

Se utilizó un diseño de bloques completos al azar (DBCA) con tres repeticiones por ambiente y para la evaluación entre los 
ambientes se realizó un análisis combinado. Para la separación de medias se efectuó la prueba de Tukey al 5\% para los factores y la interacción. Se efectuó la prueba de Shapiro-Wilks para determinar la normalidad de los datos. Para contenidos de $\mathrm{Fe}$ y $\mathrm{Zn}$ que no tuvieron una distribución normal se transformaron sus datos usando logaritmo natural (ln).

Para medir el efecto genético y ambiental sobre los contenidos de $\mathrm{Fe}, \mathrm{Zn}$ y VC, se realizó el análisis de varianza y se calculó la heredabilidad en sentido amplio $\left(\mathrm{H}^{2}\right)$ a partir del cálculo de la relación entre la varianza genética y fenotípica con la siguiente formula (Holland et al., 2003):

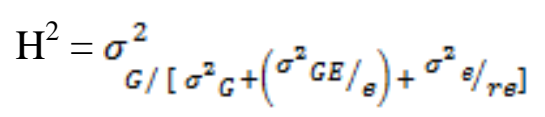

Dónde $\sigma^{2}{ }_{G}$ es el cuadrado medio de la varianza genotípica; $\sigma_{G E}^{2}$ es el cuadrado medio de la varianza de la interacción GxE; $\sigma^{2}{ }_{\theta}$ es el cuadrado medio del error; e es el número de ambientes y $\mathrm{r}$ es el número de repeticiones.

\section{Resultados y discusión}

\section{Contenido de Fe}

El análisis de varianza combinado (Tabla 2) estableció diferencias significativas para genotipos, ambientes y su interacción. El coeficiente de variación fue de $14.28 \%$ (Tabla 2) y el promedio general del contenido de $\mathrm{Fe}$ en los genotipos de 43.06 $\mu \mathrm{g} . \mathrm{g}^{-1}$ (PS) (Tabla 3).

La prueba de Tukey al 5\% para genotipos en los cuatro ambientes estableció 2 rangos de significación estadística, las variedades Superchola, INIAP-Victoria y los clones 98-38-12 y 07-32-1 se ubicaron en el primer rango con los mayores contenidos: 48.02, 47.96, 45.22 y $43.96 \mu \mathrm{g} \cdot \mathrm{g}^{-1}$ (PS) respectivamente, mientras que el clon 07 40-1 se ubicó en el último rango con el menor valor $40.01 \mu \mathrm{g} . \mathrm{g}^{-1}$ (PS) (Tabla 3).
Estos valores de $\mathrm{Fe}$ se encuentran dentro de los rangos obtenidos por Andre et al., (2007) quienes reportaron en diferentes especies de papa valores que oscilaron entre 29.87 y $157.96 \mu \mathrm{g}$. g ${ }^{-1}$ (PS). Además Burgos et al., (2007), reportaron valores entre 16.1 a $36.7 \mu \mathrm{g} . \mathrm{g}^{-1}$ (PS) de hierro en 37 accesiones proveniente de materiales diploides (S. phureja, S.stenotomum y $S$. goniocalyx), triploides ( $S$. chaucha) y tetraploide (S. tuberosum y $S$. andigena) y Brown et al., (2010) establecieron valores de 17 a $62 \mu \mathrm{g}$. $\mathrm{g}^{-1}$ (PS) de hierro en clones de mejoramiento. En los ambientes de Aláquez y Cutuglahua los genotipos mostraron mayores contenidos de $\mathrm{Fe}$ con un promedio por ambiente de $72.15 \mathrm{y}$ $57.34 \mu \mathrm{g} . \mathrm{g}^{-1}$ (PS) respectivamente (Tabla 3 ), en comparación con los ambientes de Tunshi y Chiquicha con 22.48 y $20.25 \mu \mathrm{g}$. $\mathrm{g}^{-1}$ (PS). A pesar de que la GEl fue significativa, la mayor variación fue debida al ambiente el cual aportó con el $92.45 \%$ de la variación total observada (Tabla 2). Esto probablemente se debe a las diferencias en fertilidad del suelo en los ambientes. Aláquez y Cutuglahua tuvieron los mayores contenidos de Fe (106 a 222 ppm) en suelo y también en tubérculo, mientras los suelos de Tunshi y Chiquicha presentaron valores inferiores entre 46 a 49 ppm de Fe (Anexo 1) y los tubérculos mostraron menores contenidos de $\mathrm{Fe}$. Al respecto, White et al., (2009), manifiestan que la concentración de algún elemento en el tubérculo puede deberse a las concentraciones y complejas interacciones de los elementos en el suelo, así como a la disponibilidad de los mismos.

\section{Contenido de Zinc}

El análisis de varianza para contenido de $\mathrm{Zn}$ (Tabla 2) estableció diferencias significativas para genotipos, ambientes y su interacción, con un coeficiente de variación $(\mathrm{CV})$ de $26.98 \%$. La prueba de Tukey al 5\% estableció tres rangos de significación estadística para genotipos en los cuatro ambientes. Los clones 07-40-1, 07-32-1, 07-46-8 y la variedad INIAP- 
Victoria se ubicaron en el primer rango de significación con 21.23, 18.40, $18.49 \mathrm{y}$ $21.19 \mu \mathrm{g} . \quad \mathrm{g}^{-1} \quad$ (PS) respectivamente, mientras el clon 97-25-3 se ubicó en el último rango con $13.08 \mu \mathrm{g} . \mathrm{g}^{-1}$ (PS). El promedio general de $\mathrm{Zn}$ para genotipos fue de $16.78 \mu \mathrm{g}$. $\mathrm{g}^{-1}$ (PS). Estos valores concuerdan con los rangos reportados por Brown et al., (2011) en clones de papa y por Burgos et al., (2007) en accesiones proveniente de materiales diploides $(S$. phureja, S.stenotomum y S. goniocalyx), triploides ( $S$. chaucha) y tetraploide (S.tuberosum y S. andigena), quienes reportaron valores de 12 a 18 y de 8 a 20 $\mu \mathrm{g} . \quad \mathrm{g}^{-1}$ (PS) de $\mathrm{Zn}$ respectivamente. Resultados similares fueron obtenidos por Andre et al., (2007) quienes reportan valores que oscilaron entre 12.60 y 28.83 $\mu \mathrm{g} . \mathrm{g}^{-1}$ (PS). Ereifej et al., (1998) en variedades comerciales así como Tekaling y Hammes (2005) en clones de papa, reportaron valores promedios de $20.4 \mathrm{y}$ $16.6 \mu \mathrm{g} . \mathrm{g}^{-1}$ (PS) de $\mathrm{Zn}$ respectivamente. Los ambientes de Aláquez y Cutuglahua se ubicaron en el primer rango de significación con 21.35 y $19.2483 \mu \mathrm{g}$. $\mathrm{g}^{-1}$ (PS) de Zn respectivamente (Tabla 3), seguido del ambiente de Chiquicha con $14.9983 \mu \mathrm{g} . \mathrm{g}^{-1}$ (PS) y en el último rango de significación estuvo el ambiente de Tunshi con $11.51 \mu \mathrm{g} . \mathrm{g}^{-1}$ (PS). Estas diferencias en los contenidos de Zn en los tubérculos estuvieron afectadas por el genotipo, el ambiente y su interacción. Sin embargo el ambiente aportó con la mayor variación observada $37.40 \%$ (Tabla 2).

El efecto del contenido de $\mathrm{Zn}$ en el suelo sobre los tubérculos no fue claro, los contenidos estuvieron comprendidos entre 1.20 y 7.40 ppm, En Tunshi existió una relación entre el menor contenidos de $\mathrm{Zn}$ en el suelo $(1.20 \mathrm{ppm})$ y en los tubérculos (11.51 $\mu \mathrm{g} . \mathrm{g}^{-1}$ de peso seco), pero en Aláquez ocurrió todo lo contrario con un bajo contenido de $\mathrm{Zn}$ en el suelo (1.40 ppm) se obtuvo un alto contenido en los tubérculos $\left(21.35 \mu \mathrm{g}\right.$. $\mathrm{g}^{-1}$ peso seco). Lo cual puede ser el resultado de lo discutido por White et al., (2009) y al efecto de la humedad del suelo, así en Aláquez se presentó la menor precipitación (351.2 $\mathrm{mm})$ y el mayor contenido de $\mathrm{Zn}$ en el tubérculo (21.35 $\mu \mathrm{g}$. $\mathrm{g}^{-1}$ de peso seco), mientras en Cutuglahua, Chiquicha y Tunshi con precipitaciones de 717.40, 618.90 y $364.00 \mathrm{~mm}$ mostraron menores contenidos de $\mathrm{Zn}$ en los tubérculos (Anexo 2).

\section{Contenido de Vitamina C}

El análisis de varianza combinado para VC (Tabla 2) estableció diferencias significativas para genotipos, ambientes y su interacción, con un CV de $15.97 \%$ y un promedio general $84.23 \mathrm{mg}$. $100 \mathrm{~g}^{-1}$ (PF). La prueba de Tukey al 5\% para genotipos (Tabla 4) estableció tres rangos de significación. Los clones 07-24-18, 07-32$15,07-40-1$ y 07-28-2 se ubicaron en el primer rango con 98.42, 94.00, $92.75 \mathrm{y}$ $91.83 \mathrm{mg} .100 \mathrm{~g}^{-1}$ (PF) respectivamente, mientras que la variedad INIAP-Fripapa $y$ el clon 97-25-3 se ubicaron en el último rango con el menor contenido $72.92 \mathrm{y}$ $72.33 \mathrm{mg}$. $100 \mathrm{~g}^{-1}$ (PF). En Tunshi $\mathrm{y}$ Aláquez los contenidos de VC se ubicaron en el primer rango de significación con 95.83 y $93.10 \mathrm{mg} .100 \mathrm{~g}^{-1}$ (PF), seguido de Chiquicha con $79.43 \mathrm{mg}$. $100 \mathrm{~g}^{-1}$ (PF) mientras Cutuglahua se ubicó en el último rango con $68.57 \mathrm{mg}$. $100 \mathrm{~g}^{-1}(\mathrm{PF})$. Estudios previos reportan valores de $\mathrm{VC}$ en base fresca entre 6.5 y $36.9 \mathrm{mg} .100 \mathrm{~g}^{-1} \mathrm{PF}$ en variedades nativas del CIP (Burgos et al., 2009), de 8.4 a $20.1 \mathrm{mg} .100 \mathrm{~g}^{-1} \mathrm{PF}$ para variedades Noruegas (Nordbotten et al., 2000); de 8.8 a 24.1 mg. $100 \mathrm{~g}^{-1}$ PF para variedades Canadienses (Mullin et al., 1991) y de 16 a $46 \mathrm{mg} .100 \mathrm{~g}^{-1} \mathrm{PF}$ para variedades Coreanas (Han et al., 2004).

Estos resultados muestran una gran variación para los contenidos de VC de los genotipos evaluados, los valores reportados son más altos que los estudios descritos.

$\mathrm{El} \mathrm{pH}$ pudo afectar el contenido de VC, en Cutuglahua se obtuvo el menor contenido y su pH 5.79 en comparación con Aláquez, Chiquicha y Tunshi que presentaron mayores contenidos de $\mathrm{VC}$ con 
pH mayor a 7.38 (Anexo 1). Pascale et al., (2001) encontraron un mayor contenido de $\mathrm{VC}$ en tomate asociado con un alto $\mathrm{pH}$ en el suelo. Burgos et al., (2009) obtuvieron mayores contenidos de VC en papas nativas en suelos con $\mathrm{pH}$ de 7.6 en comparación con otros ambientes con menor $\mathrm{pH}(<5.2)$. La fertilidad del suelo fue otro de los factores que pudo afectar a los contenidos de VC. El contenido de materia orgánica en los ambientes estuvo entre 1.70 a $8.80 \%$, los contenidos de nitrógeno $(\mathrm{N})$, fósforo $(\mathrm{P})$ y potasio $(\mathrm{K})$ que estuvieron entre 33 a $61 \mathrm{ppm}, 26$ a 233 ppm y 0.59 a 1.70 meq. $100 \mathrm{ml}^{-1}$ respectivamente (Anexo 1). $\mathrm{La}$ concentración de VC estuvieron influenciadas significativamente por el ambiente y su interacción las cuales contribuyeron con 29.16 y $27.43 \%$ de la variación total observada respectivamente en comparación con el genotipo (14.13\%) (Tabla 2). 
Tabla 2. Análisis de varianza combinado y porcentaje de la variación total (\%) en los contenidos de Fe, Zn, VC y RT (t.ha ${ }^{-1}$ ) en genotipos de papa (Solanum sp.) para la evaluación del efecto GEI.

\begin{tabular}{|c|c|c|c|c|c|c|c|c|c|}
\hline \multirow{2}{*}{$\begin{array}{l}\text { Fuente de } \\
\text { variación }\end{array}$} & \multirow{2}{*}{$\begin{array}{c}\text { Grados } \\
\text { Libertad }\end{array}$} & \multicolumn{4}{|c|}{ Cuadrados Medios } & \multicolumn{4}{|c|}{ Proporción de la Variación Total (\%) } \\
\hline & & $\mathrm{Fe}^{\mathrm{a}}$ & $\mathbf{Z n}^{\mathbf{a}}$ & $\overline{V C}$ & RT & $\mathbf{F e}$ & $\mathbf{Z n}$ & $\mathrm{VC}$ & RT \\
\hline Total & 167 & 0.34 & 0.13 & 415.23 & 168.99 & & & & \\
\hline Repetición & 2 & $4.7 \mathrm{E}-03^{\mathrm{ns}}$ & $2.7 \mathrm{E}-03^{\mathrm{ns}}$ & $196.12^{\mathrm{ns}}$ & $5.28^{\mathrm{ns}}$ & 0.02 & 0.05 & 0.57 & 0.04 \\
\hline Genotipos (G) & 13 & $0.04^{*}$ & $0.15^{* *}$ & $753.78^{* *}$ & $383.97^{* *}$ & 0.88 & 9.15 & 14.13 & 17.69 \\
\hline Ambiente (E) & 3 & $17.46^{* *}$ & $2.66^{* *}$ & $6740.64^{* *}$ & $5536.45^{* *}$ & 92.45 & 37.40 & 29.16 & 58.85 \\
\hline $\mathrm{G} \times \mathrm{E}$ & 39 & $0.06^{* * *}$ & $0.16^{* *}$ & $487.65^{* *}$ & $77.81^{* *}$ & 3.88 & 29.99 & 27.43 & 10.75 \\
\hline Error & 110 & 0.01 & 0.05 & 181.02 & 32.51 & 2.77 & 23.46 & 28.72 & 12.67 \\
\hline $\mathrm{CV}(\%)^{\mathrm{a}}$ & & 3.32 & 7.76 & & & & & & \\
\hline $\mathrm{CV}(\%)$ & & 14.28 & 26.98 & 15.97 & 15.13 & & & & \\
\hline
\end{tabular}


Tabla 3. Prueba de Tukey al $5 \%$ para contenido de Fe y Zn ( $\mu \mathrm{g} . \mathrm{g}^{-1}$ de peso seco) en genotipos de papa (Solanum $s p$.) para la evaluación del efecto GEI.

\begin{tabular}{|c|c|c|c|c|c|c|c|c|c|c|c|}
\hline \multirow[t]{2}{*}{ Genotipos } & \multicolumn{5}{|c|}{ Hierro } & \multicolumn{6}{|c|}{ Zinc } \\
\hline & Chiquicha & Tunshi & Cutuglahua & Alaquez & $\mathrm{PG}$ & Chiquicha & Tunshi & Cutuglahua & Alaquez & PG & \\
\hline 07-24-18 & $19.97 \mathrm{abc} \pm 2 . \mathrm{z}$ & $20.13 \mathrm{ab} \pm 0.99$ & $58.40 a b c \pm 1.10$ & $63.67 \pm 0.40$ & $40.54 \mathrm{ab} \pm 21.52^{* *}$ & $15.80 \pm 2.21$ & $11.83 \pm 4.99$ & $17.80 \mathrm{bcd} \pm 3.60$ & $14.73 \mathrm{~b} \pm 2.40^{1}$ & $15.04 \mathrm{abc}^{3} \pm 3.73^{2}$ & $\mathrm{~ns}$ \\
\hline 07-28-2 & $20.73 \mathrm{abc} \pm 1.9$ & $23.73 \mathrm{ab} \pm 1.25$ & $47.33 \mathrm{c} \pm 2.86$ & $70.07 \pm 3.44$ & $40.47 \mathrm{ab} \pm 20.95 * *$ & $16.73 \pm 1.40$ & $13.20 \pm 2.52$ & $20.60 \mathrm{abcd} \pm 4.39$ & $16.00 \mathrm{~b} \pm 3.00$ & $16.63 \mathrm{abc} \pm 3.78$ & $\mathrm{~ns}$ \\
\hline 07-32-1 & $22.40 \mathrm{ab} \pm 1.13$ & $28.23 \mathrm{a} \pm 4.12$ & $54.93 \mathrm{bc} \pm 6.04$ & $70.27 \pm 3.11$ & $43.96 \mathrm{a} \pm 20.68 * *$ & $16.60 \pm 1.49$ & $11.13 \pm 1.63$ & $27.10 \mathrm{ab} \pm 0.87$ & $18.77 \mathrm{~b} \pm 4.10$ & $18.40 \mathrm{ab} \pm 6.33$ & $* *$ \\
\hline 07-32-15 & $20.80 \mathrm{abc} \pm 0.9$ & $22.97 \mathrm{ab} \pm 4.41$ & $47.93 \mathrm{c} \pm 2.18$ & $72.13 \pm 8.27$ & $40.96 \mathrm{ab} \pm 22.24 * *$ & $16.53 \pm 3.04$ & $12.03 \pm 3.58$ & $16.53 \mathrm{bcd} \pm 2.16$ & $17.20 \mathrm{~b} \pm 1.20$ & $15.58 \mathrm{abc} \pm 3.12$ & $\mathrm{~ns}$ \\
\hline 07-40-1 & $18.77 \mathrm{abc} \pm 2.9$ & $22.80 \mathrm{ab} \pm 3.30$ & $45.77 \mathrm{c} \pm 6.20$ & $72.70 \pm 3.05$ & $40.01 \mathrm{~b} \pm 22.73 * *$ & $12.50 \pm 0.87$ & $11.90 \pm 2.80$ & $15.93 \mathrm{bcd} \pm 1.46$ & $44.57 \mathrm{a} \pm 11.81$ & $21.23 \mathrm{ab} \pm 15.1$ & $* *$ \\
\hline $07-46-8$ & $18.07 \mathrm{abc} \pm 2.5$ & $23.40 \mathrm{ab} \pm 4.62$ & $46.57 \mathrm{c} \pm 6.58$ & $86.73 \pm 16.47$ & $43.69 \mathrm{ab} \pm 29.34^{* *}$ & $16.17 \pm 0.64$ & $12.47 \pm 0.85$ & $14.03 \mathrm{~cd} \pm 1.67$ & $31.30 \mathrm{ab} \pm 19.80$ & $18.49 \mathrm{abc} \pm 11.6$ & ** \\
\hline $97-25-3$ & $21.10 \mathrm{abc} \pm 3.7$ & $24.17 \mathrm{ab} \pm 2.20$ & $54.63 \mathrm{bc} \pm 9.26$ & $71.27 \pm 1.39$ & $42.79 \mathrm{ab} \pm 22.40 * *$ & $14.30 \pm 1.87$ & $9.77 \pm 0.31$ & $14.30 \mathrm{~cd} \pm 0.53$ & $13.93 b \pm 0.85$ & $13.08 \mathrm{c} \pm 2.2$ & $\mathrm{~ns}$ \\
\hline $98-2-6$ & $20.50 \mathrm{abc} \pm 2.3$ & $23.17 \mathrm{ab} \pm 4.12$ & $48.40 \mathrm{c} \pm 1.30$ & $74.43 \pm 5.86$ & $41.63 \mathrm{ab} \pm 23.05^{* *}$ & $13.53 \pm 1.29$ & $9.77 \pm 1.42$ & $13.03 \mathrm{~d} \pm 3.09$ & $26.93 \mathrm{ab} \pm 2.85$ & $15.82 \mathrm{abc} \pm 7.15$ & ** \\
\hline 98-38-12 & $24.10 \mathrm{a} \pm 1.06$ & $27.23 \mathrm{a} \pm 4.99$ & $51.63 b c \pm 6.24$ & $77.90 \pm 15.23$ & 45.22 a $\pm 23.80 * *$ & $15.20 \pm 1.91$ & $14.73 \pm 1.83$ & $16.37 \mathrm{bcd} \pm 1.16$ & $23.83 \mathrm{ab} \pm 4.25$ & $17.53 a b c \pm 4.43$ & $\mathrm{~ns}$ \\
\hline INIAP-Estela & 24.23 a 1.14 & $19.70 a b \pm 2.29$ & $61.23 \mathrm{abc} \pm 7.49$ & $70.37 \pm 3.17$ & $43.88 \mathrm{ab} \pm 23.48^{* *}$ & $14.47 \pm 1.56$ & $10.33 \pm 3.02$ & $13.83 \mathrm{~d} \pm 0.67$ & $19.43 b \pm 1.04$ & $14.52 b c \pm 3.72$ & $\mathrm{~ns}$ \\
\hline INIAP-Fripapa & $16.53 \mathrm{c} \pm 1.64$ & $16.80 \mathrm{~b} \pm 0.36$ & $64.13 \mathrm{abc} \pm 3.46$ & $67.03 \pm 4.84$ & $41.13 \mathrm{ab} \pm 25.7(* *$ & $12.43 \pm 2.41$ & $8.73 \pm 1.21$ & $26.07 \mathrm{abc} \pm 10.38$ & $15.70 \mathrm{~b} \pm 2.10$ & $15.73 \mathrm{abc} 8.2$ & ** \\
\hline INIAP-Natividad & $18.37 \mathrm{abc} \pm 1.6$ & $19.57 \mathrm{ab} \pm 1.53$ & $62.40 \mathrm{abc} \pm 14.65$ & $69.77 \pm 2.35$ & $42.53 \mathrm{ab} \pm 25.57 * *$ & $17.13 \pm 3.55$ & $13.47 \pm 4.10$ & $20.13 \mathrm{abcd} \pm 6.12$ & $15.60 \mathrm{~b} \pm 0.35$ & $16.58 \mathrm{abc} \pm 4.32$ & $\mathrm{~ns}$ \\
\hline INIAP-Victoria & $17.60 \mathrm{bc} \pm 1.91$ & $21.93 \mathrm{ab} \pm 3.65$ & $85.23 \mathrm{a} \pm 12.01$ & $67.07 \pm 3.56$ & $47.96 \mathrm{a} \pm 30.76 * *$ & $14.47 \pm 1.79$ & $11.77 \pm 2.25$ & $32.23 \mathrm{a} \pm 6.30$ & $26.30 \mathrm{ab} \pm 8.80$ & $21.19 \mathrm{a} \pm 9.99$ & $* *$ \\
\hline Superchola & $20.30 \mathrm{abc} \pm 1.0$ & $20.90 a b \pm 0.72$ & $74.13 \mathrm{ab} \pm 16.78$ & $76.73 \pm 17.00$ & $48.02 \mathrm{a} \pm 30.41 * *$ & $14.03 \pm 4.18$ & $10.03 \pm 1.50$ & $21.43 \mathrm{abcd} \pm 6.89$ & $14.67 \mathrm{~b} \pm 2.17$ & $15.04 b c \pm 5.6$ & ns \\
\hline PL & $20.25 d \pm 2.79$ & $22.48 c \pm 3.93$ & $57.34 b \pm 13.13$ & $72.15 \mathrm{a} \pm 8.83$ & 43.06 & $14.99 \mathrm{~b} \pm 2.39$ & $11.51 \mathrm{c} \pm 2.70$ & $19.24 \mathrm{a} \pm 6.75$ & $21.35 \mathrm{a} \pm 10.18$ & 16.78 & \\
\hline
\end{tabular}

${ }^{1}$ Valores promedios \pm desviación estándar $(\mathrm{n}=3)$

${ }^{2}$ Valores promedios \pm desviación estándar $(\mathrm{n}=12)$

${ }^{3}$ Letras diferentes indican diferencias significativas entre genotipos según la prueba de Tukey al $5 \%$

** = Significativo al $1 \%$ entre ambientes

ns $=$ No significativo entre ambientes, $\mathrm{PG}=$ Promedio general entre ambientes, $\mathrm{PL}=$ Promedio por ambiente 


\section{Rendimiento}

El análisis de varianza combinado para rendimiento (Tabla 2) estableció diferencias significativas para genotipo, ambiente y su interacción, con un CV de $15.13 \%$ y un promedio general del rendimiento de los clones y variedades de 37.68 t. ha ${ }^{-1}$ (Tabla 4). La prueba de Tukey al 5\% para genotipos estableció seis rangos de significación, donde las variedades INIAP-Victoria e INIAP-Fripapa, así como el clon 98-2-6 se ubicaron en el primer rango de significación con 46.47 , 43.37 y 43.78 t. ha $^{-1}$ respectivamente. El clon 07-40-1 se ubicó en el último rango de significación con $27.18 \mathrm{t}$. ha ${ }^{-1}$. Los rendimientos promedios en los ambientes de Chiquicha, Tunshi y Cutuglahua fueron superiores a $40 \mathrm{t}$. ha ${ }^{-1}$, mientras en Aláquez se obtuvo el menor rendimiento con 20.57 t. ha ${ }^{-1}$ (Tabla 4). El ambiente afectó significativamente al rendimiento, el cual aportó con el $58.85 \%$ de la variación total observada (Tabla 2), valor menor al reportado por Gutiérrez y Muñoz (2009), quienes reportan que el factor ambiente fue determinante en el rendimiento y este aportó con el $80.4 \%$ de la variación total observada.

La cantidad de precipitación en cada ambiente fue diferente, lo que se reflejó en el rendimiento, en Aláquez durante el ciclo del cultivo su precipitación fue de $351.2 \mathrm{~mm}$ con un rendimiento promedio menor a 20.60 t. ha $^{-1}$, mientras en Cutuglahua, Chiquicha y Tunshi con precipitaciones de 717.4, 610.9 y 364.0 mm respectivamente, mostraron rendimientos superiores a 40 t. ha ${ }^{-1}$. Cabe indicar que en Tunshi la precipitación fue baja $(364.0 \mathrm{~mm})$. Sin embargo, este ambiente disponía de riego lo cual complementó el requerimiento de agua del cultivo (Anexo 2). 
Tabla 4. Prueba de Tukey al $5 \%$ para vitamina C (mg. $\left.100 \mathrm{~g}^{-1} \mathrm{PF}\right)$ y rendimiento (t.ha $\left.{ }^{-1}\right)$ en genotipos de papa (Solanum tuberosum) para la evaluación del efecto GEI.

\begin{tabular}{|c|c|c|c|c|c|c|c|c|c|c|c|c|}
\hline \multirow[t]{2}{*}{ Genotipos } & \multicolumn{5}{|c|}{ Vitamina $\mathbf{C}$} & \multicolumn{7}{|c|}{ Rendimiento } \\
\hline & Chiquicha & Tunshi & Cutuglahua & Alaquez & PG & & Chiquicha & Tunshi & Cutuglahua & Alaquez & PG & \\
\hline $07-24-18$ & $82.33 \mathrm{ab} \pm 4.04$ & $107.33 \mathrm{abc} \pm 1.53$ & $82.67 \mathrm{ab} \pm 12.58$ & $121.33 a \pm 46.32$ & $98.42 \mathrm{a} \pm 26.93$ & $\mathrm{~ns}$ & $48.87 \mathrm{ab} \pm 5.34$ & $43.90 \mathrm{bcd} \pm 5.70$ & $38.70 \mathrm{abc} \pm 6.79$ & $21.60 \mathrm{abc} \pm 4.68^{1}$ & $38.27 \mathrm{bcd}^{3} \pm 11.77^{2}$ & $* *$ \\
\hline $07-28-2$ & $85.67 \mathrm{ab} \pm 5.13$ & $101.67 \mathrm{abcd} \pm 7.37$ & $86.00 \mathrm{ab} \pm 7.21$ & $94.00 \mathrm{ab} \pm 16.64$ & $91.83 \mathrm{ab} \pm 11.04$ & $\mathrm{~ns}$ & $45.20 \mathrm{ab} \pm 3.5$ & $46.70 \mathrm{abc} \pm 5.63$ & $35.10 a b c \pm 7.14$ & $18.00 \mathrm{abc} \pm 4.12$ & 36.25 bcde \pm 12.78 & $* *$ \\
\hline $07-32-1$ & $84.67 \mathrm{ab} \pm 2.89$ & $109.33 \mathrm{ab} \pm 4.16$ & $52.67 \mathrm{ab} \pm 4.04$ & $81.67 \mathrm{ab} \pm 9.29$ & $82.08 \mathrm{abc} \pm 21.53$ & $* *$ & $38.27 \mathrm{ab} \pm 3.6$ & $34.67 \mathrm{~cd} \pm 3.10$ & $29.70 \mathrm{bc} \pm 2.70$ & $12.60 \mathrm{c} \pm 4.12$ & 28.81 ef \pm 10.69 & $* *$ \\
\hline $07-32-15$ & $85.33 \mathrm{ab} \pm 10.5$ & $99.00 \mathrm{abcd} \pm 14.80$ & $71.00 \mathrm{ab} \pm 1.73$ & $120.67 \mathrm{a} \pm 22.59$ & $94.00 \mathrm{ab} \pm 22.78$ & *** & $33.60 \mathrm{ab} \pm 8.1$ & $44.77 \mathrm{bcd} \pm 4.57$ & $43.20 \mathrm{abc} \pm 5.40$ & $19.80 \mathrm{abc} \pm 5.62$ & $35.34 \mathrm{cde} \pm 11.60$ & ** \\
\hline $07-40-1$ & $93.33 \mathrm{a} \pm 9.87$ & $122.00 \mathrm{a} \pm 5.00$ & $61.67 \mathrm{ab} \pm 10.02$ & $94.00 \mathrm{ab} \pm 9.54$ & $92.75 \mathrm{ab} \pm 23.55$ & $* *$ & $33.10 \mathrm{~b} \pm 4.47$ & $31.50 \mathrm{~d} \pm 6.56$ & $30.60 a b c \pm 6.24$ & $13.50 \mathrm{bc} \pm 0.00$ & $27.18 \mathrm{f} \pm 9.35$ & ** \\
\hline $07-46-8$ & $76.33 \mathrm{ab} \pm 4.93$ & $71.00 \mathrm{~cd} \pm 6.08$ & $68.67 a b \pm 19.5$ & $90.33 \mathrm{ab} \pm 24.58$ & $76.58 \mathrm{bc} \pm 16.35$ & ns & $39.87 \mathrm{ab} \pm 2.4$ & $40.23 \mathrm{bcd} \pm 1.72$ & $27.90 c \pm 7.79$ & $15.30 b c \pm 3.12$ & $30.83 \operatorname{def} \pm 11.36$ & ** \\
\hline $97-25-3$ & $66.67 \mathrm{~b} \pm 5.13$ & $65.00 \mathrm{~d} \pm 5.57$ & $51.00 \mathrm{~b} \pm 1.0$ & $106.67 \mathrm{ab} \pm 10.12$ & $72.33 c \pm 22.32$ & $* *$ & $44.37 \mathrm{ab} \pm 3.41$ & $37.80 \mathrm{~cd} \pm 3.06$ & $53.10 \mathrm{a} \pm 10.91$ & $23.40 \mathrm{abc} \pm 4.12$ & $39.67 \mathrm{abc} \pm 12.53$ & ** \\
\hline $98-2-6$ & $86.00 \mathrm{ab} \pm 4.36$ & $107.00 \mathrm{abc} \pm 18.19$ & $57.67 \mathrm{ab} \pm 19.5$ & $86.67 \mathrm{ab} \pm 11.24$ & $84.33 a b c \pm 22.18$ & $* *$ & $50.07 \mathrm{ab} \pm 3.5$ & $52.13 \mathrm{ab} \pm 1.44$ & $51.30 \mathrm{ab} \pm 2.70$ & $21.60 \mathrm{abc} \pm 5.40$ & $43.78 \mathrm{ab} \pm 13.73$ & ** \\
\hline $98-38-12$ & $72.00 \mathrm{ab} \pm 8.54$ & $89.00 \mathrm{abcd} \pm 25.24$ & $60.33 \mathrm{ab} \pm 17.9$ & $100.67 a b \pm 6.03$ & $80.50 \mathrm{abc} \pm 21.33$ & $\mathrm{~ns}$ & $50.07 \mathrm{ab} \pm 3.5$ & $48.23 \mathrm{abc} \pm 4.01$ & $34.20 \mathrm{abc} \pm 6.79$ & $15.30 \mathrm{bc} \pm 3.12$ & $36.95 \mathrm{bcd} \pm 15.06$ & $* *$ \\
\hline INIAP-Estela & $67.33 \mathrm{~b} \pm 9.45$ & $106.00 \mathrm{abc} \pm 10.58$ & $58.33 \mathrm{ab} \pm 13.8$ & $91.33 \mathrm{ab} \pm 6.51$ & $80.75 a b c \pm 21.67$ & $* *$ & $45.37 \mathrm{ab} \pm 9.3^{\prime}$ & $47.53 \mathrm{abc} \pm 2.83$ & $50.40 \mathrm{abc} \pm 9.48$ & $21.60 \mathrm{abc} \pm 0.00$ & $41.23 \mathrm{abc} \pm 13.32$ & $* *$ \\
\hline INIAP-Fripapa & $71.67 \mathrm{ab} \pm 2.31$ & $82.00 \mathrm{bcd} \pm 9.00$ & $73.33 \mathrm{ab} \pm 24.58$ & $64.67 \mathrm{~b} \pm 17.47$ & $72.92 \mathrm{c} \pm 14.92$ & ns & $45.03 \mathrm{ab} \pm 9.1$ & $52.83 \mathrm{ab} \pm 5.00$ & $53.10 \mathrm{a} \pm 13.59$ & $22.50 \mathrm{abc} \pm 4.12$ & $43.37 \mathrm{ab} \pm 15.04$ & $* *$ \\
\hline INIAP-Natividad & $73.00 \mathrm{ab} \pm 13.1$ & $86.00 \mathrm{abcd} \pm 11.00$ & $75.00 \mathrm{ab} \pm 8.19$ & $88.00 \mathrm{ab} \pm 9.54$ & $80.50 \mathrm{abc} \pm 11.37$ & $\mathrm{~ns}$ & $51.13 \mathrm{a} \pm 7.95$ & $39.37 \mathrm{bcd} \pm 4.57$ & $38.70 \mathrm{abc} \pm 4.12$ & $28.80 \mathrm{a} \pm 5.62$ & $39.5 \mathrm{abc} \pm 9.61$ & $* *$ \\
\hline INIAP-Victoria & $91.00 \mathrm{a} \pm 6.24$ & $104.33 \mathrm{abc} \pm 16.80$ & $66.67 \mathrm{ab} \pm 10.97$ & $73.67 a b \pm 7.23$ & $83.92 \mathrm{abc} \pm 18.08$ & $* *$ & $48.40 \mathrm{ab} \pm 3.0$ & $59.17 \mathrm{a} \pm 8.50$ & $53.10 \mathrm{a} \pm 5.62$ & $25.20 \mathrm{ab} \pm 1.56$ & $46.47 \mathrm{a} \pm 14.19$ & ** \\
\hline Superchola & $76.67 \mathrm{ab} \pm 1.15$ & $92.00 \mathrm{abcd} \pm 9.54$ & $95.00 \mathrm{a} \pm 16.52$ & $89.67 a b \pm 16.29$ & $88.33 \mathrm{abc} \pm 12.96$ & $\mathrm{~ns}$ & $40.87 \mathrm{ab} \pm 6.9$ & $46.80 \mathrm{abc} \pm 3.13$ & $43.20 \mathrm{abc} \pm 7.14$ & $28.80 \mathrm{a} \pm 5.62$ & $39.92 a b c \pm 8.68$ & $* *$ \\
\hline PL & $79.43 \mathrm{~b} \pm 10.25$ & $95.83 \mathrm{a} \pm 18.39$ & $68.57 \mathrm{c} \pm 17.01$ & $93.10 \mathrm{a} \pm 21.58$ & 84.23 & & $43.87 \mathrm{a} \pm 7.53$ & $44.69 \mathrm{a} \pm 8.27$ & $41.59 \mathrm{a} \pm 11.01$ & $20.57 \mathrm{~b} \pm 6.08$ & 37.68 & \\
\hline
\end{tabular}

${ }^{1}$ Valores promedios \pm desviación estándar $(\mathrm{n}=3),{ }^{2}$ Valores promedios \pm desviación estándar $(\mathrm{n}=12)$

${ }^{3}$ Letras diferentes indican diferencias significativas entre genotipos según la prueba de Tukey al $5 \%$, ** =Significativo al $5 \%$ entre ambientes

$\mathrm{PF}=$ Peso fresco, $\mathrm{PG}=$ Promedio general entre ambientes, $\mathrm{PL}=$ Promedio por ambiente 


\section{Heredabilidad en sentido amplio $\left(\mathbf{H}^{2}\right)$.}

La heredabilidad en sentido amplio para $\mathrm{Fe} \quad \mathrm{y} \quad \mathrm{Zn}$ fueron de 0.32 y 0.20 respectivamente (Tabla 5), valor inferior a los reportados por Brown et al., (2010) y (2011) quienes obtuvieron valores entre 0.64 a 0.76 . El valor de $\mathrm{H}^{2}$ para $\mathrm{VC}$ fue de 0.35; otras investigaciones como las de Salas et al., (2012) reportaron estimados de $\mathrm{H}^{2}$ entre 0.93 y 0.71 respectivamente. El valor de $\mathrm{H}^{2}$ para rendimiento $\left(\mathrm{t} \mathrm{ha}^{-1}\right)$ fue de 0.80 valor inferior a lo reportado por Javier et al., (1974) con 0.93 y superior a lo reportado por Pérez et al., (2006) y (2007) con 0.44 y 0.78 respectivamente. Los valores de $\mathrm{H}^{2}$ para $\mathrm{Fe}, \mathrm{Zn}$ y VC fueron inferiores a 0.50 lo que muestra menor efecto genético y mayor efecto del ambiente. Las condiciones edafoclimáticas fueron muy diferentes en cada ambiente, la precipitación $\mathrm{y}$ temperatura fluctuaron entre 351.2 a $717.4 \mathrm{~mm}$ y 12 a $17^{\circ} \mathrm{C}$ de temperatura promedio por ciclo del cultivo respectivamente (Anexo 2). La fertilidad de los suelos fue muy heterogénea, esto se evidencia en los contenidos de N (33-61 ppm), $\mathrm{P}$ (26-233 ppm), K (0.59-1.70 meq.100 $\left.\mathrm{ml}^{-1}\right)$, materia orgánica $(1.70-$ $8.80 \%)$ y micronutrientes como Fe (106$222 \mathrm{ppm})$ y Zn (1.20- $7.40 \mathrm{ppm}$ ) (Anexo 1), lo cual influyó significativamente en los contenidos de $\mathrm{Fe}, \mathrm{Zn}, \mathrm{VC}$ en los tubérculos.

Tabla 5. Heredabilidad en sentido amplio para las variables para contenido de $\mathrm{Fe}, \mathrm{Zn}$ y VC en genotipos de papa para la evaluación del efecto GEI.

\begin{tabular}{|c|c|c|c|c|}
\hline & $\mathrm{Fe}$ & $\mathrm{Zn}$ & Vitamina $\mathrm{C}$ & $\begin{array}{c}\text { Rendimiento } \\
\text { t.ha }^{-1}\end{array}$ \\
\hline \multicolumn{5}{|c|}{ Componentes de Varianza } \\
\hline$\sigma_{a}^{2}$ & 37.79 & 20.48 & 181.02 & 32.51 \\
\hline$\sigma_{G}^{2}$ & -5.79 & -1.82 & 22.18 & 25.51 \\
\hline$\sigma_{G E}^{2}$ & 37.67 & 23.16 & 102.21 & 15.10 \\
\hline \multicolumn{5}{|c|}{ Heredabilidad en sentido amplio $\left(H^{2}\right)$} \\
\hline $\mathrm{H}^{2}$ & 0.32 & 0.20 & 0.35 & 0.80 \\
\hline
\end{tabular}

\section{Conclusiones}

Existió gran variación para los diferentes contenidos evaluados en los genotipos de papa, así como para el rendimiento total. Lo cual fue el resultado de un efecto significativo del genotipo, el ambiente y su interacción, donde la mayor variación fue efecto del ambiente.

La mayor variación del ambiente fue resultado de las variaciones en la cantidad de precipitación combinado con los contenidos variables de macro, micro nutrientes, materia orgánica y el $\mathrm{pH}$ del suelo de las diferentes localidades.

La concentración de Fe en los tubérculos estuvo asociada con el contenido de este elemento en el suelo, mientras que para el $\mathrm{Zn}$ existieron otros factores como la humedad y el $\mathrm{pH}$ que probablemente afectaron la disponibilidad de este elemento en el suelo.

La gran variación observada para los contenidos de $\mathrm{Fe}$ y VC y su $\mathrm{H}^{2}$ intermedia, nos indica que es posible a través de mejoramiento genético incrementar los contenidos de estos elementos. Mientras que para aumentar $\mathrm{Zn}$ esta no sería la mejor estrategia.

\section{Conflictos de Intereses}

Esta investigación fue desarrollada por el Instituto Nacional de Investigaciones Agropecuarias (INIAP) y los autores 
declaran no tener conflictos con la publicación de este trabajo de investigación.

\section{Agradecimientos}

Al Proyecto Fortalecimiento de la innovación agrícola pro-pobre para la seguridad alimentaria en la región andina (CIP-ISSANDES). Financiamiento de la Comisión de la Unión Europea a través del Fondo Internacional de Desarrollo Agrícola (FIDA). Al Proyecto Fortalecimiento de la investigación y la producción de papa para la seguridad alimentaria de las familias de la sierra ecuatoriana. Financiamiento de la Secretaria Nacional de Planificación y Desarrollo (SENPLADES).

\section{Referencias citadas}

Andre, C.M.; Ghislain, M.; Bertin P.; Mouhssin, O.; del Rosario Herrera, M.; Hoffmann L.; Hausman J.F.; Larondelle Y.; Evers D. 2007. Andean potato cultivars (Solanum tuberosum L.) as a source of antioxidant and mineral micronutrients. Journal of Agricultural and Food Chemistry 55: 366-378.

Bonierbale, M.; Amoros, W.; Salas, E.; Cáceres, M. 2008. Valor agregado y nutricional de la papa nativa. En Memoria III Congreso Iberoamericano de la Patata. Vitoria Gasteiz, España. p. 191.

Brown, C.R.; Haynes, K.G.; Moore, M.; Pavek, M.J.; Hane, D.C.; Love, S.L.; Novy, R.G.; Miller, J.C. 2010. Stability and broad-sense heritability of mineral content in potato: Iron. American Journal of Potato Research 87:390-396.

Brown, C.R.; Haynes, K.G.; Moore, M.; Pavek, M.J.; Hane, D.C.; Love, S.L.; Novy, R.G.; Miller, J.C. 2011. Stability and broad-sense heritability of mineral content in potato: Zinc. American Journal of Potato Research 88:238-244.

Burgos, G.; Amoros, W.; Morote, M.; Stangoulis, J.; Bonierbale, M. 2007. Iron and zinc concentration of native andean potato varieties from a human nutrition perspective. Journal of the Science of Food and Agriculture. 87 (4): 668-675.

Burgos, G.; Auqui, S.; Amoros, W.; Salas, E.; Bonierbale, M. 2009. Ascorbic acid concentration of native Andean potato varieties as affected by environment, cooking and storage. Journal of Food Composition and Analysis. 22 (2009) 533538.

Ereifej, K. I.; Shibli, R. A.; Ajlouni, M. M.; Hussein, A. 1998. Mineral contents of whole tubers and selected tissues of ten potato cultivars grown in Jordan. J. Food Sci. Technol. 35:55-58.

Falconer, D. S.; Mackay, T. F. S. 1996. Introduction to Quantitative Genetics, Fourth Edition. Ed. Longman, England. $464 \mathrm{p}$.

Gutiérrez, A.; Muñoz J. 2009. Interacción genotipo por ambiente de siete variedades de papa en la zona papera de Chirquí, Panama. Revista Latinoamericana de la Papa. 15(1):12-19.

Haase, N. U. 2008. Healthy aspects of potatoes as part of human diet. Potato Research. 51:239-258.

Han, J.; Kosukue, N.; Young, K.; Lee, K.; Friedman, M. 2004 Distribution of ascorbic acid in potato tubers and in homeprocessed and commercial potato foods. J.Agri. Food Chem. 52 (21):6516-6521.

Holland, J. B.; Nyquist, W. E.; CervantesMartinez, C. T. 2003. Estimating and interpreting heritability for plant breeding: An update. Plant Breeding Reviews 22: 9112.

Javier, T.; Molina G.; Casas D. 1974. Correlaciones genéticas e índices de selección en la genotecnia de la papa (Solanum tuberosum L.). Agrociencia 16:21-37.

Love, S. L.; Pavek, J. J. 2008. Positioning the potato as a primary food source of vitamin C. Am. J. Potato Res. 85:277-285. 
Mullin, W.J.; Jui, P.Y.; Nadeau, L.; Smyril, T.G. 1991. The vitamin C content of seven cultivars of potatoes grown across Canada. Can. Inst. Food Sci. Techonl. J. Indian Potato Assoc. 12(34): 158-164.

Nordbotten, A.; Loken, B.; Rimestad, H. 2000. Sampling of potatoes to determine representative values for nutrient content in a national food composition table. J. Food Comp. Annual. 13:369-377.

Pascale, S.; de Maggio, A.; Fogliano, V.; Ambrosino, P.; Ritienia. 2001. Irrigation with saline water improves carotenoids content and antioxidant activity of tomato. J. Hortic. Sci. Biothecnol. 76(4):447-453.

Pérez, D.; Corral A.; Miningo, M.; Gonzalez A. 2006. Estudio preliminar de diez cruzas del Centro Internacional de la Papa (CIP). Revista Ciencias Agrícolas. 3$12 \mathrm{pp}$.

Pérez, D.; Vásquez, L.; Sahagún, J.; Rivera, A. 2007.Variabilidad y caracterización de diez variedades de papa en tres localidades del Estado de México. Revista Chapingo. 13(1)13-19.
Salas, E.; Montañez, A.; Juarez, H.; Amoros, W.; Burgos, G.; Canto, R.; Scurrah, M.; De Haan, S.; Bonierbale, M. 2012. Stability analysis for nutritional contents (minerals and vitamin c) on 40 native potato cultivars. En memoria XXV Congreso de la Asociación Latinoamericana de la papa. Uberlandia, Brasil.

Storey, M. 2007. The harvested crop. In: Potato Biology and Biotechnology, Advances and Perspectives. (Vreugdenhil, D., Ed.). Elsevier, Amsterdam, The Netherlands. 441-470.

Tekaling, T.; Hammes, P.S. 2005. Growth and productivity of potato as influenced by cultivar and reproductive growth. II. Growth analysis, tuber yield and quality. Sci. Hort. 105:29-44.

White, P., Bradsahw, J., Finlay, M., Dale, B., Ramsay, G., Hammond, J., Broadley, M. 2009. Relationships between yield and mineral concentrations in potato tubers HortScience $\quad 44$ (1):6-10. 
Anexo 1. Reporte resumido de los análisis de suelos de los ambientes cultivo para la evaluación de la interacción GEI sobre los contenidos de vitamina C hierro y zinc en cuatro ambientes.

\begin{tabular}{llllll}
\hline Descripción & Unidad & $\begin{array}{l}\text { Pichincha } \\
\text { Cutuglahua }\end{array}$ & $\begin{array}{l}\text { Cotopaxi } \\
\text { Aláquez }\end{array}$ & $\begin{array}{l}\text { Tungurahua } \\
\text { Chiquicha }\end{array}$ & $\begin{array}{l}\text { Chimborazo } \\
\text { Tunshi }\end{array}$ \\
\hline $\mathrm{N}$ & $\mathrm{ppm}$ & 48.00 & 41.00 & 61.00 & 33.00 \\
$\mathrm{P}$ & $\mathrm{ppm}$ & 46.00 & 78.00 & 233.00 & 26.00 \\
$\mathrm{~K}$ & $\mathrm{meq} / 100 \mathrm{ml}$ & 0.59 & 0.90 & 1.70 & 0.78 \\
$\mathrm{Fe}$ & $\mathrm{ppm}$ & 222.00 & 106.00 & 46.00 & 49.00 \\
$\mathrm{Zn}$ & $\mathrm{ppm}$ & 3.50 & 1.40 & 7.40 & 1.20 \\
$\mathrm{MO}$ & $\%$ & 8.80 & 3.70 & 2.70 & 1.70 \\
$\mathrm{pH}$ & & 5.79 & 7.60 & 8.26 & 7.38 \\
\hline
\end{tabular}

Fuente: Análisis de suelo del Laboratorio de Manejo de Suelos

y Aguas de la EESC del INIAP

Anexo 2. Precipitación y temperatura durante el ciclo de cultivo para la evaluación de la interacción GEI para contenidos de vitamina C hierro y zinc en cuatro ambientes.

\begin{tabular}{llll}
\hline Provincia & Ambiente & $\begin{array}{l}\text { Precipitación } \\
\text { mm / ciclo del }\end{array}$ & Temperatura \\
& & cultivo & \\
\hline Pichincha & Cutuglahua & $717.4^{1}$ & $12.2^{1}$ \\
Cotopaxi & Aláquez & $351.2^{2}$ & $13.2^{2}$ \\
Tungurahua & Chiquicha & $610.9^{3}$ & $13.7^{3}$ \\
Chimborazo & Tunshi & $364.0^{3}$ & $16.63^{3}$ \\
\hline 1 & Estación meteorológica del Instituto Nacional Autónomo de Investigaciones Agropecuarias (INIAP) Santa Catalina. \\
2ireción General de Aviación Civil. Departamento de Meteorología Aeronáutica. Sección Climatología. \\
3 Estaciones Experimentales Guazlán y Patate (Estación Guadalupe)
\end{tabular}

